\title{
Screening for Cancer in Patients with Acute Venous Thromboembolic Disease ${ }^{\#}$
}

\author{
Marc Blondon ${ }^{1}$ \\ ${ }^{1}$ Division of Angiology and Hemostasis, Faculty of Medicine, Geneva \\ University Hospitals and Faculty of Medicine, Genève, Switzerland \\ Hämostaseologie 2021;41:42-47.
}

Address for correspondence Marc Blondon, MD, MS, Division of Angiology and Hemostasis, Hôpitaux Universitaires de Genève, 4 Rue Gabrielle-Perret-Gentil, 1205 Genève, Switzerland (e-mail: marc.blondon@hcuge.ch).

\begin{abstract}
Keywords

- venous thrombosis

- cancer

- pulmonary embolism

- diagnosis

Active cancer causes approximately $25 \%$ of all acute events of venous thromboembolism (VTE). While most of the cancer diagnoses are known or clinically apparent at the time of VTE, care providers and patients may be worried about the 3 to $8 \%$ risk of occult cancer occurring in the year after VTE. Several studies have compared limited to extensive cancer screening after acute VTE, especially with the addition of abdominal computed tomography (CT) or whole-body PET-CT, with the hope to shorten the time to cancer diagnosis and lead to less advanced cancer stages. These studies have not shown improved clinical outcomes with an extensive screening, and have led to current recommendations of limited screening for cancer in patients with acute VTE, including unprovoked cases. Several risk assessment models have been developed to identify patients at greatest risk of occult cancer, however, with low discriminative performances and no current clinical usefulness. Some clinical situations may empirically deserve a more thorough cancer screening, such as unprovoked upper extremity deep vein thrombosis (DVT), bilateral leg DVT, descending leg DVT, or recurrent VTE during anticoagulation.
\end{abstract}

Case scenario: A nonsmoking 56-year-old man without any relevant medical history is treated for an acute unprovoked pulmonary embolism as an outpatient. A thorough medical history and physical examination reveal no symptoms or signs suggestive of an occult cancer. Which cancer screening procedures are recommended in this situation?

Venous thromboembolism (VTE) is a common vascular disease with multiple known genetic and acquired risk factors. Cancer has long been recognized as both an important acquired cause of VTE and a marker of poor prognosis from VTE, with particularly high risks of recurrent VTE despite anticoagulation and of bleeding. ${ }^{1}$ An important question for both patients and care providers at the time of acute VTE is whether a yet undiagnosed cancer may have played a role. Such undiagnosed occult cancers are active neoplastic diseases, which are not

\footnotetext{
\# Invited review for the 65th Annual Meeting of the Society of Thrombosis and Hemostasis Research (GTH 2021).
}

received

November 8, 2020

accepted after revision

December 17, 2020 readily diagnosed or clinically obvious at the time of VTE diagnosis, and have been defined by most studies as cancer diagnosed more than 30 days after a deep vein thrombosis (DVT) or pulmonary embolism (PE).

In this narrative review, we will evaluate the current evidence to guide the use of cancer screening procedures at the time of acute VTE, through the following five questions: (1) What are the risks and types of cancer, in patients with acute VTE? (2) What have shown the studies comparing different levels of cancer screening? (3) Are there risk factors or risk assessment models to personalize screening strategies? (4) Which specific situations may trigger an extensive screening strategy? and (5) What do the recommendations currently advise?

\section{What Are the Risks and Types of Cancer, in Patients with Acute VTE?}

About one in four patients with acute VTE suffers from (mostly) diagnosed, or yet undiagnosed, cancer. (c) 2021. Thieme. All rights reserved. Georg Thieme Verlag KG,

Rüdigerstraße 14,

70469 Stuttgart, Germany
DOI https://doi.org/ 10.1055/a-1339-7328. ISSN 0720-9355. 
A clinically relevant increase in the incidence of occult cancer has long been recognized after an acute VTE. In the large international RIETE registry of acute VTE, among more than 50,000 symptomatic confirmed VTE patients, $17 \%$ had a known cancer and $4 \%$ were diagnosed with cancer within 30 days; among 5,863 patients with a 24-month follow-up and no baseline cancer, $8 \%$ suffered from an occult cancer. Similarly, in a meta-analytic effort of more than 2,000 patients with unprovoked VTE followed up prospectively for at least 12 months, 5 to $6 \%$ of patients were diagnosed with cancer after acute VTE. ${ }^{2}$ Two-thirds of cancers were diagnosed during the early screening period, and one-third during follow-up. This estimate of 5 to $6 \%$, which arises from studies published between 2010 and 2016, contrasts with a previously estimate of $10 \%$ from studies published before $2008 .^{3}$ This decrease in the estimated risk of occult cancer over time may be due to a possibly lower methodological quality of older studies. Another explanation may be a change of population, due to the overdiagnosis of VTE with the advent of computed tomographic (CT) scan diagnosis. ${ }^{4}$ Nevertheless, the contemporary estimate of 5 to $6 \%$ is likely more valid.

Therefore, the aims of extensive screening procedures are (1) the early diagnosis of occult cancer in 3 to $8 \%$ of patients (best estimate of $5.2 \%)^{2}$ and (2) the improvement of clinical outcomes following these diagnoses.

Most research has focused on cancer screening after unprovoked VTE, because the risk to develop cancer is much greater after unprovoked $(7.6 \%)$ than provoked VTE (1.9\%). ${ }^{3}$ Nevertheless, about one-third of occult cancer was diagnosed in RIETE participants with risk factors for VTE, such as a recent surgery or immobility. ${ }^{5}$ In the HOKUSAI-VTE study, the risk of occult cancer was similar between unprovoked (1.8\%) and provoked (2.1\%) VTE. ${ }^{6}$

The distribution of cancer type differs slightly between the general population and patients with acute VTE, highlighting differential risks of VTE between cancer types. While breast and prostate cancer are the two most incident cancer in women and men, lung cancer is most commonly associated with acute VTE ( - Table 1 ). In the contemporary international GARFIELD-VTE registry, lung, colorectal, breast, gynecological, prostate, urological, and lymphoma account for two-thirds of all acute VTE cancer. ${ }^{7}$ What is actually most relevant for screening is the distribution of occult cancer among acute VTE patient. In RIETE, occult cancer remained again versatile in its localization, closely resembling the distribution described earlier. Among 444 participants with a diagnosis of cancer beyond 30 days after acute VTE, most suffered from lung $(16 \%$, especially men), colorectal (15\%), prostate (10\%), hematological (7\%), bladder (6\%), stomach (5\%), breast (5\%), and pancreas (5\%) cancers. ${ }^{5}$ The high VTE risk associated with stomach, pancreas, brain, and hematological malignancies explains their increased preponderance after acute VTE, compared with the general population.

This wide variety of localization of both solid and hematological tumors among patients with acute VTE makes screening for cancer challenging and limits the utility of screening to a localized organ.
Table 1 The 10 cancers with the highest incidence in the general population (2008-2012 Swiss data) ${ }^{28}$ and with the greatest prevalence among patients with VTE (GARFIELD-VTE registry)

\begin{tabular}{|l|l|l|}
\hline General population & VTE patients $^{7}$ \\
\hline Women & Men & All \\
\hline Breast & Prostate & Lung \\
\hline Colorectal & Lung & Colorectal \\
\hline Lung & Colorectal & Breast \\
\hline Melanoma & Melanoma & Gynecological \\
\hline Uterus & Bladder & Lymphoma \\
\hline $\begin{array}{l}\text { Non-Hodgkin's } \\
\text { lymphoma }\end{array}$ & Non-Hodgkin's & Urological \\
\hline Pancreas & ENT & \\
\hline Ovaries & Kidney & $\begin{array}{l}\text { Upper } \\
\text { gastrointestinal }\end{array}$ \\
\hline Thyroid & Pancreas & Pancreas \\
\hline Leukemia & Liver & Myeloma \\
\hline
\end{tabular}

Abbreviations: VTE, venous thromboembolism; ENT, ear nose and throat.

Table 2 Screening procedures tested in interventional studies, compared with a limited screening

\begin{tabular}{|l|l|}
\hline $\begin{array}{l}\text { Procedure (on top a routine screening } \\
\text { based on history and physical exam, } \\
\text { basic laboratory, chest X-ray, and age- } \\
\text { and sex-directed cancer screening) }\end{array}$ & $\begin{array}{l}\text { Finding and } \\
\text { reference }\end{array}$ \\
\hline $\begin{array}{l}\text { CT of the abdomen and pelvis, including } \\
\text { virtual colonoscopy and gastroscopy }\end{array}$ & Not useful $^{9}$ \\
\hline Whole-body ${ }^{18}$ F-FDG PET-CT & Possibly useful $^{10}$ \\
\hline $\begin{array}{l}\text { CT of the chest, abdomen, and pelvis } \\
\text { with fecal occult blood test }\end{array}$ & Not useful $^{11}$ \\
\hline
\end{tabular}

\section{What Have Shown the Studies Comparing Different Levels of Cancer Screening?}

Several controlled studies have compared different screening strategies for occult cancer after acute VTE (-Table 2). Most have focused on abdominal imaging, following the observation that abdominal-pelvic imaging was the single most effective procedure to increase the proportion of occult cancer detection. $^{3}$

The first clinical trial (SOMIT), and the only positive one, was conducted in several European centers 25 years ago in 201 participants. $^{8}$ Due to a peculiar study design, participants of the control group were not informed of study participation and no guidance was provided for cancer screening. In contrast, the extensive screening group underwent extremely comprehensive procedures, including an abdominal ultrasound and CT, a gastroscopy, a colonoscopy or sigmoidoscopy, sputum cytology, blood tumor biomarkers, and a mammography/Pap smear for women and a 
transabdominal ultrasound of the prostate/prostate-specific antigen (PSA) for men. At 2 years, 9.8 to $14.1 \%$ of participants were diagnosed with cancer. The extensive screening identified $93 \%$ of cancer within 40 days of acute VTE, while no cancer was identified early in the control group. This finding supportive for an aggressive screening strategy is, however, tempered by the premature stop of this study (at $20 \%$ of the anticipated sample) and particularly by the surprising lack of any diagnosis in the control group, suggesting subpar medical care not representative of today's clinical care.

The Canadian SOME trial randomized, in an open-label format, 854 patients with unprovoked VTE to limited screening or a limited screening plus CT strategies. ${ }^{9}$ The limited screening already included a complete history and physical exam; blood analyses (blood count, liver function testing); a chest X-ray; and, when indicated, a mammography, a pelvic exam, and Pap testing (in sexually active women) and a PSA and prostate examination (in men $>40$ years). The limited screening plus CT added a CT of the abdomen and pelvis, including a virtual colonoscopy and gastroscopy. At 1 year, 3.2 to $4.5 \%$ of participants had a diagnosis of occult cancer, but there was no difference between strategies with regard to the proportion of occult cancer detected early (74\% with vs. $71 \%$ without the $\mathrm{CT}$ ), the time to cancer diagnosis, or the cancer-related mortality.

A third clinical trial, the open-label French MVTEP study, randomized 394 patients with acute unprovoked VTE to a limited screening strategy (similar to the SOME study) or to a limited screening plus whole-body ${ }^{18}$ F-FDG PET-CT. ${ }^{10}$ At 2 years, 6.1 to $6.6 \%$ of participants had a diagnosis of cancer. The strategy with PET-CT detected more cancer (in 5.6\% of participants, $92 \%$ of all cancer diagnoses during the study) than the strategy without PET-CT (in $2.0 \%$ of participants, $31 \%$ of all cancer diagnoses during the study), but the findings of the primary analysis remained statistically borderline $(p=0.07)$, with a theoretical number needed to examine of 27 to detect one additional cancer. While PET-CT cannot be recommended based on this study, final conclusions of the utility of PET-CT await the conduct of several ongoing clinical trials, either larger or in more selected groups of patients.

A fourth clinical trial, open-label and based in Italy, randomized 195 participants with unprovoked VTE to a standard screening strategy (routine laboratory, chest Xray, mammography, and additional investigations guided by signs/symptoms) or a CT-based strategy with the addition of CT scan of the chest, abdomen, and pelvis and fecal occult blood test. ${ }^{11}$ At 2 years, 10.3 to $12.2 \%$ of participants had a diagnosis of cancer, without difference in the proportion diagnosed during the initial strategy: $80 \%$ by the standard screening strategy and $83 \%$ by the CT-based strategy.

Albeit not randomized, an observational Dutch study compared centers that offered a limited screening (history, physical exam, blood laboratory, and chest X-ray) with centers that offered an extensive screening (limited screening + thoracoabdominal CT scan + mammography). ${ }^{12}$ After a median of 2.5 years, 7.3 to $8.8 \%$ were diagnosed with cancer: 60 and 33\% of them during early screening process in the extensive and limited screening groups, respectively. The risk of occult cancer after the screening procedure was, however, not different between both groups, nor was the overall mortality or cancer-related mortality, and the study was stopped due to futility.

Overall, findings of these studies do not support an extensive screening for occult cancer after acute VTE. The only positive study was the older Italian trial comparing an extensive and standardized screening to really no screening at all, which does not represent current standard of care. While the number of cancer detected through an initial extensive screening procedure exceeds that of a limited screening procedure, the risk of occult cancer after the initial screening is not different, without evidence of more limited or treatable tumors in patients with an extensive screening strategy. ${ }^{3}$ In contrast, the key message from these studies is that a limited screening workup identifies the vast majority of occult cancer, without the need for thorough imaging in routine care. Furthermore, there is a potential for greater diagnostic tests and invasive procedures for false-positive signals in case of extensive screening. ${ }^{13}$

\section{Are There Risk Factors or Risk Assessment Models to Personalize Screening Strategies?}

Without any doubt, the most important predictor of occult cancer is an advanced age, following the age distribution of cancer in the general population: the risk of occult cancer is much lower in patients younger than 50 years (1\%) than in patients older than 50 years $(6.7 \%){ }^{2}$ Other risk factors for occult VTE have not been consistently demonstrated in various data and remain more presumptive. The risk of occult cancer does not differ by the initial location of VTE (PE, DVT, both), even when considering abdominal tumors. ${ }^{14}$

Extensive screening strategies, while non-efficient in all patients after unprovoked VTE, may yield better results in selected patients with a higher risk of occult cancer. Two main risk models have been created to predict individuals in whom cancer is diagnosed more than 30 days after the diagnosis of VTE, that is, for whom cancer is not evident at the time of acute VTE ( - Table 3 ).

Table 3 Risk assessment models developed to identify patients at high risk of occult cancer

\begin{tabular}{|l|l|}
\hline RIETE model & SOME model \\
\hline Male sex $[+1]$ & Age $\geq 60$ y $[+1]$ \\
\hline Age $>70$ y [+2] & Current smoking [+1] \\
\hline Chronic lung disease [+1] & Previous VTE $[+1]$ \\
\hline Anemia [+2 $]^{\text {a }}$ & \\
\hline Platelet count $>350 \mathrm{G} / \mathrm{L}[+1]$ & \\
\hline Postsurgery VTE $[-2]$ & \\
\hline Previous VTE $[-1]$ & \\
\hline Low risk $\leq 2$ & Low risk $\leq 1$ \\
\hline High risk $\geq 3$ & High risk $\geq 2$ \\
\hline
\end{tabular}

Abbreviation: VTE, venous thromboembolism.

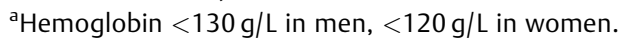


The RIETE risk model was developed and validated in this large registry data, from unprovoked and provoked VTE ( $n=444$ VTE patients with occult cancer vs. 5,417 without occult cancer). The strongest predictor of occult cancer was advanced age ( $>70$ years) and other risk factors were male sex, chronic lung disease (in the validation cohort replaced by active smoking), anemia, and a high platelet count. Protective factors were a postsurgical VTE and a prior VTE. The SOME risk model was abstracted from the SOME RCT (33 occult cancer vs. 821 none), which included only unprovoked VTE. It identified advanced age ( $\geq 60$ years), active smoking, and a prior provoked VTE as risk factors for occult cancer. Interestingly, only advanced age and smoking are found in both scores, with contrasting results on the influence of prior VTE.

Both scores underwent a parallel evaluation in the HOKUSAI-VTE randomized trial, where their performance was disappointing, with a poor discrimination and sensitivity (218 occult cancers among 8,032 acute VTE patients). For both scores, the high-risk and low-risk groups had 2.7 to $2.9 \%$ and 1.7 to $1.8 \%$ risks of occult VTE at 2 years, respectively, and most participants with occult cancer were categorized as low risk. ${ }^{6}$ One must remember that the anticipated selection of a healthy sample in this randomized trial may also affect the overall risk of occult at 2 years, and caution must be kept when interpreting these results.

Another parallel evaluation was done in an individual patient data meta-analysis of the three studies described earlier (the Trousseau study, the MVTEP trial, and the SOME trial), yielding 98 occult cancers among 1,830 patients, and somewhat better results. ${ }^{15}$ For the RIETE score, risks of occult cancer were 5.9 and 2.9 in the high- and low-risk groups, respectively, with a sensitivity of $40 \%$. For the SOME score, risks of occult cancer were 5.0 and $3.8 \%$ in the high- and lowrisk groups, respectively, with a sensitivity of $22 \%$.

Taken together, these results do not support the use of these risk assessment models to guide differential screening strategies, given the lack of clinically relevant risk discrimination, and the fact that most occult cancers occur in patients not deemed at high risk by these scores. Research projects are ongoing to improve the prediction of occult cancer after VTE. For example, much attention and excitement is given to the development of blood-based liquid biopsy, and RNA of tumor-educated platelets may be a potential biomarker of interest for early cancer detection. The interaction and infiltration of circulating platelets with tumor tissue may result in specific splice events of platelet RNA and inclusion of circulating RNA, which may be detected by sequencing platforms. ${ }^{16}$ The utility of such biomarkers for clinical prediction of occult cancer after VTE is currently investigated in a multicentric prospective cohort (NCT02739867).

\section{Which specific situations may trigger an extensive screening strategy?}

In spite of the previously described null findings for an extensive screening, some specific clinical situations may deserve a special screening attention, although this is based more on clinical experience than published evidence.
Upper extremity deep vein thrombosis (UEDVT) is more prevalent among patients with cancer than without cancer, in particular with the presence of a central venous catheter. ${ }^{7}$ When occurring without venous lines and known cancer, the occurrence of a proximal UEDVT should trigger further imaging to explore the possibility of a local venous compression, whether it is related to cancer or not (thoracic outlet syndrome for example).

The occurrence of bilateral DVT merits some discussion. More than 20 years ago, a signal of a specific risk of underlying neoplasm in this situation was already documented ${ }^{17}$ : among this sample of 80 patients, the prevalence of known cancer at the time of DVT was $20 \%$, and $16 \%$ of patients without known cancer, but a bilateral DVT received a diagnosis of cancer within the hospital stay. These findings were replicated by two French cohorts, with documented risks of occult cancer between 10 and $26 \%$ after bilateral DVT. ${ }^{18,19}$

Although DVT and PE are not differentially associated with occult cancer, this may be different for descending proximal leg DVT. The natural history of leg DVT is an ascending thrombus, originating in the veins of the calf, and extending upward toward the iliofemoral veins. A descending thrombus, which originates in the iliac veins and descends toward the popliteal vein, suggests an abdominal compression, and should motivate a precise abdominopelvic imaging in search for a solid tumor, an adenopathy or nontumoral processes such as a congenital or acquired May-Turner anomaly.

Other clinical situations that may suggest an underlying neoplastic activity include recurrent VTE, in particular when occurring despite anticoagulation, ${ }^{1}$ recurrent unprovoked superficial vein thrombosis occurring on nonvaricose veins, and concurrent arterial and venous thrombi.

The role of cancer in splanchnic vein thrombosis (SVT; portal, mesenteric, splenic, and suprahepatic veins) also deserves some comment, although hepatic cirrhosis remains the number 1 cause. In an international registry based in tertiary hospitals, solid cancers accounted for about a fourth of SVT. ${ }^{20}$ The vast majority of tumors is of digestive location, with a preponderance of hepatocellular carcinoma, colon, pancreas, and gastric tumors. ${ }^{21}$ Furthermore, in the 5 years after a diagnosis of SVT, solid tumors (in particular hepatocellular carcinoma and pancreas cancer) are diagnosed in approximately $15 \%$ of patients, corresponding to a fourfold relative increase compared with the general population. ${ }^{22}$ Myeloproliferative neoplasms cause approximately $8 \%$ of SVT, and are particularly associated with Budd-Chiari syndrome (thrombosis of the suprahepatic veins), as highlighted by a $40 \%$ prevalence of the JAK2V617F mutation in such cases. ${ }^{23}$ In spite of the lack of randomized trials evaluating the role of cancer screening in SVT without cirrhosis, a diagnosis of abdominal cancer should be sought and after excluding abdominal diseases, myeloproliferative neoplasms should be considered.

\section{What do the recommendations currently advise?}

In light of the described recent evidence, the SSC of the International Society on Thrombosis and Hemostasis has published a guidance document on the topic of cancer 
Table 4 Guidance information from societies

\begin{tabular}{|l|l|}
\hline Society & Recommendation \\
\hline $\begin{array}{l}\text { International Society } \\
\text { Haemostasis (2017) }\end{array}$ & $\begin{array}{l}\text { Patients with unprovoked VTE } \\
\text { should undergo limited cancer } \\
\text { screening, including a thorough } \\
\text { medical history and physical ex- } \\
\text { amination, laboratory investiga- } \\
\text { tions (complete blood count, } \\
\text { calcium, urinalysis, and liver } \\
\text { function tests), and chest X-ray. } \\
\text { Age-specific and gender-specific } \\
\text { cancer screening (colon, breast, } \\
\text { cervix, and prostate) should also } \\
\text { be performed according to na- } \\
\text { tional recommendations. } \\
\text { Routine cancer screening in } \\
\text { patients with provoked VTE is } \\
\text { not recommended }\end{array}$ \\
\hline $\begin{array}{l}\text { European Society for } \\
\text { Medical Oncology } \\
\text { (2011) }\end{array}$ & $\begin{array}{l}\text { Patients with unprovoked VTE } \\
\text { should undergo only physical } \\
\text { examination, occult fecal blood } \\
\text { test, chest X-ray, urological visit } \\
\text { in men and gynecological visit } \\
\text { in women. More extensive } \\
\text { examinations addressed in case } \\
\text { of a strong clinical suspicion of } \\
\text { occult cancer [II, C] }\end{array}$ \\
\hline lence (2020) & $\begin{array}{l}\text { In patients with unprovoked } \\
\text { VTE, review the medical history } \\
\text { and baseline blood test results } \\
\text { and offer a physical examina- } \\
\text { tion. Do not offer further } \\
\text { investigations for cancer to } \\
\text { people with unprovoked VTE } \\
\text { unless they have relevant } \\
\text { clinical symptoms or signs }\end{array}$ \\
\hline
\end{tabular}

Abbreviation: VTE, venous thromboembolism.

screening (-Table 4). ${ }^{24}$ Very simply, it suggests to undergo limited cancer screening (medical history, physical examination, laboratory measurements, and chest X-ray) as well as age-specific and gender-specific cancer screening. The use of PET-CT or abdominopelvic CT is therefore not recommended. A similar guidance has been provided by the United Kingdom National Institute for Health and Care Excellence in 2020, ${ }^{25}$ with the suggestion to restrict to medical history, physical examination, and baseline blood test for investigation of cancer in patients with unprovoked acute VTE. Furthermore, the NICE document highlights the non-cost-effectiveness of extensive screening interventions. ${ }^{26}$ Older, albeit similar recommendations were also provided by the European Society for Medical Oncology. ${ }^{27}$

The current guidance on age-specific and gender-specific cancer screening is delineated in -Table 5 , based on the United States Preventive Services Task Force.

\section{Conclusions}

Despite an important research effort, an extensive screening for occult cancer has not been shown to improve clinical outcomes
Table 5 Summary of age-specific and sex-specific cancer screening procedures in the general population, based on the United States Preventive Services Task Force

\begin{tabular}{|l|l|l|}
\hline Population & $\begin{array}{l}\text { Screened } \\
\text { cancer }\end{array}$ & $\begin{array}{l}\text { Procedure } \\
\text { (recommendation } \\
\text { grade) }\end{array}$ \\
\hline Women, 50-74 y & $\begin{array}{l}\text { Breast } \\
\text { cancer }\end{array}$ & $\begin{array}{l}\text { Mammography } \\
\text { every 2 y (B) }\end{array}$ \\
\hline Women, 40-49 y & $\begin{array}{l}\text { Breast } \\
\text { cancer }\end{array}$ & $\begin{array}{l}\text { Screening } \\
\text { mammography } \\
\text { based on individual } \\
\text { discussion (C) }\end{array}$ \\
\hline Women, 21-65 y & $\begin{array}{l}\text { Cervical } \\
\text { cancer }\end{array}$ & $\begin{array}{l}\text { Cervical Pap test } \\
\text { and/or HPV test } \\
\text { every 3 y (A) }\end{array}$ \\
\hline All, 50-75 y & $\begin{array}{l}\text { Colorectal } \\
\text { cancer }\end{array}$ & $\begin{array}{l}\text { Fecal occult blood } \\
\text { annually, or colo- } \\
\text { noscopy every 10 y, } \\
\text { or sigmoidoscopy } \\
\text { every 5 y (A) }\end{array}$ \\
\hline $\begin{array}{l}\text { All, 76-85 y } \\
\text { Active smokers or } \\
\text { recent quitters } \\
\text { year smoking, } \\
\mathbf{5 5 - 8 0} \text { y }\end{array}$ & $\begin{array}{l}\text { Colorectal } \\
\text { cancer }\end{array}$ & $\begin{array}{l}\text { Screening for } \\
\text { colorectal cancer } \\
\text { based on individual } \\
\text { discussions (C) }\end{array}$ \\
\hline Men, 55-69 y & $\begin{array}{l}\text { Prostate } \\
\text { cancer }\end{array}$ & $\begin{array}{l}\text { Low-dose lung scan } \\
\text { every year (B) } \\
\text { ing for prostate } \\
\text { cancer based on } \\
\text { individual } \\
\text { discussions (C) }\end{array}$ \\
\hline
\end{tabular}

Abbreviations: HPV, human papillomavirus; PSA, prostate-specific antigen.

after acute VTE, and is currently discouraged. Ongoing trials may change this current recommendation, particularly in subgroups of patients deemed at higher risk of occult cancer or in studies with bigger sample sizes exploring the usefulness of PET-CT (NCT04304651; NCT03907583). For the time being, care providers are advised to implement the evidence-based recommendations of limited, but cautious, screening procedures.

Resolution of the case scenario: The 56-year-old man with an acute PE had undergone a thoracic angio-CT for the diagnosis of PE, which did not show any lesion suggestive of cancer. A limited-screening strategy with a thorough history, physical examination, and basic blood tests is performed, which only reveals an iron-deficient anemia (hemoglobin: $108 \mathrm{~g} / \mathrm{L}$ ). This anemia leads to the conduct of a normal colonoscopy (also indicated by the age of the patient), a gastroscopy showing celiac disease without dysplasia, and the exclusion of a multiple myeloma by protein immunoelectrophoresis. Two years after the acute PE, this patient is doing well, without any diagnosis of occult cancer.

The authors declare that they have no conflict of interest. 


\section{References}

1 Prandoni P, Lensing AWA, Piccioli A, et al. Recurrent venous thromboembolism and bleeding complications during anticoagulant treatment in patients with cancer and venous thrombosis. Blood 2002;100(10):3484-3488

2 van Es N, Le Gal G, Otten H-M, et al. Screening for occult cancer in patients with unprovoked venous thromboembolism: a systematic review and meta-analysis of individual patient data. Ann Intern Med 2017;167(06):410-417

3 Carrier M, Le Gal G, Wells PS, Fergusson D, Ramsay T, Rodger MA. Systematic review: the Trousseau syndrome revisited: should we screen extensively for cancer in patients with venous thromboembolism? Ann Intern Med 2008;149(05):323-333

4 Wiener RS, Schwartz LM, Woloshin S. Time trends in pulmonary embolism in the United States: evidence of overdiagnosis. Arch Intern Med 2011;171(09):831-837

5 Jara-Palomares L, Otero R, Jiménez D, et al; RIETE Investigators. Development of a risk prediction score for occult cancer in patients with VTE. Chest 2017;151(03):564-571

6 Kraaijpoel N, van Es N, Raskob GE, et al. Risk scores for occult cancer in patients with venous thromboembolism: a post hoc analysis of the Hokusai-VTE study. Thromb Haemost 2018;118 (07):1270-1278

7 Weitz JI, Haas S, Ageno W, et al; GARFIELD-VTE Investigators. Cancer associated thrombosis in everyday practice: perspectives from GARFIELD-VTE. J Thromb Thrombolysis 2020;50(02):267-277

8 Piccioli A, Lensing AWA, Prins MH, et al; SOMIT Investigators Group. Extensive screening for occult malignant disease in idiopathic venous thromboembolism: a prospective randomized clinical trial. J Thromb Haemost 2004;2(06):884-889

9 Carrier M, Lazo-Langner A, Shivakumar S, et al; SOME Investigators. Screening for occult cancer in unprovoked venous thromboembolism. N Engl J Med 2015;373(08):697-704

10 Robin P, Le Roux P-Y, Planquette B, et al; MVTEP Study Group. Limited screening with versus without (18)F-fluorodeoxyglucose PET/CT for occult malignancy in unprovoked venous thromboembolism: an open-label randomised controlled trial. Lancet Oncol 2016;17(02):193-199

11 Prandoni P, Bernardi E, Valle FD, et al. Extensive computed tomography versus limited screening for detection of occult cancer in unprovoked venous thromboembolism: a multicenter, controlled, randomized clinical trial. Semin Thromb Hemost 2016;42(08):884-890

12 Van Doormaal FF, Terpstra W, Van Der Griend R, et al. Is extensive screening for cancer in idiopathic venous thromboembolism warranted? J Thromb Haemost 2011;9(01):79-84

13 Robin P, Le Roux P-Y, Le Moigne E, et al. Additional testing following screening strategies for occult malignancy diagnosis in patients with unprovoked venous thromboembolism. Thromb Res 2017;155:6-9

14 Jara-Palomares L, van Es N, Praena-Fernandez JM, et al. Relationship between type of unprovoked venous thromboembolism and cancer location: an individual patient data meta-analysis. Thromb Res 2019;176:79-84

15 Mulder FI, Carrier M, van Doormaal F, et al. Risk scores for occult cancer in patients with unprovoked venous thromboembolism: results from an individual patient data meta-analysis. J Thromb Haemost 2020;18(10):2622-2628

16 Wurdinger T, In't Veld SGJG, Best MG. Platelet RNA as pan-tumor biomarker for cancer detection. Cancer Res 2020;80(07): 1371-1373

17 Rance A, Emmerich J, Guedj C, Fiessinger JN. Occult cancer in patients with bilateral deep-vein thrombosis. Lancet 1997;350 (9089):1448-1449

18 Seinturier C, Bosson J-L, Colonna M, Imbert B, Carpentier PH. Site and clinical outcome of deep vein thrombosis of the lower limbs: an epidemiological study. J Thromb Haemost 2005;3(07): 1362-1367

19 Bura A, Cailleux N, Bienvenu B, et al. Incidence and prognosis of cancer associated with bilateral venous thrombosis: a prospective study of 103 patients. J Thromb Haemost 2004;2(03):441-444

20 Ageno W, Riva N, Schulman S, et al. Long-term clinical outcomes of splanchnic vein thrombosis: results of an international registry. JAMA Intern Med 2015;175(09):1474-1480

21 Valeriani E, Di Nisio M, Riva N, et al; International Registry on Splanchnic Vein Thrombosis (IRSVT) Study Group. Clinical history of cancer-associated splanchnic vein thrombosis. J Thromb Haemost 2020;(epub ahead of print). Doi: 10.1111/jth.15214

22 Søgaard KK, Farkas DK, Pedersen L, Sørensen HT. Splanchnic venous thrombosis is a marker of cancer and a prognostic factor for cancer survival. Blood 2015;126(08):957-963

23 Smalberg JH, Arends LR, Valla DC, Kiladjian J-J, Janssen HLA, Leebeek FWG. Myeloproliferative neoplasms in Budd-Chiari syndrome and portal vein thrombosis: a meta-analysis. Blood 2012; 120(25):4921-4928

24 Delluc A, Antic D, Lecumberri R, Ay C, Meyer G, Carrier M. Occult cancer screening in patients with venous thromboembolism: guidance from the SSC of the ISTH. J Thromb Haemost 2017;15 (10):2076-2079

25 National Institute for Health and Care Excellence (NICE) Venous thromboembolic diseases: diagnosis, management and thrombophilia testing. November 2020:1-47

26 Coyle K, Carrier M, Lazo-Langner A, et al. Cost effectiveness of the addition of a comprehensive CT scan to the abdomen and pelvis for the detection of cancer after unprovoked venous thromboembolism. Thromb Res 2017;151:67-71

27 Mandalà M, Falanga A, Roila FESMO Guidelines Working Group. Management of venous thromboembolism (VTE) in cancer patients: ESMO Clinical Practice Guidelines. Ann Oncol 2011;22 (Suppl 6):vi85-vi92

28 OFSP data. Accessed Nov 2, 2020 at: https://www.bag.admin.ch/ bag/fr/home/zahlen-und-statistiken/zahlen-fakten-nichtuebertragbare-krankheiten/krebserkrankungen-schweiz.html 